\title{
Guaranteed Cost Finite-Time Control for Positive Switched Linear Systems with Time-Varying Delays
}

\author{
Xiangyang Cao, Leipo Liu, Zhumu Fu, Xiaona Song, and Shuzhong Song \\ College of Information Engineering, Henan University of Science and Technology, Luoyang 471023, China \\ Correspondence should be addressed to Leipo Liu; liuleipo123@163.com
}

Received 21 September 2016; Accepted 16 January 2017; Published 19 February 2017

Academic Editor: Ai-Guo Wu

Copyright (c) 2017 Xiangyang Cao et al. This is an open access article distributed under the Creative Commons Attribution License, which permits unrestricted use, distribution, and reproduction in any medium, provided the original work is properly cited.

\begin{abstract}
This paper considers the guaranteed cost finite-time control for positive switched linear systems with time-varying delays. The definition of guaranteed cost finite-time boundedness is firstly given. Then, by using the mode-dependent average dwell time approach, a static output feedback law and a state feedback control law are constructed, respectively, and sufficient conditions are obtained to guarantee that the closed-loop system is guaranteed cost finite-time boundedness. Such conditions can be easily solved by linear programming. Finally, an example is given to illustrate the effectiveness of the proposed method.
\end{abstract}

\section{Introduction}

Positive switched systems are a class of hybrid systems consisting of a family of positive subsystems and a switching law that specifies which subsystem will be activated along the system trajectory at each instant of time. In recent years, many remarkable results about the problems of stability analysis and controller synthesis of positive switched systems have been presented [1-8]. Among these existing results, the average dwell time (ADT) approach and mode-dependent average dwell time (MDADT) technique are widely used; the latter allows that every subsystem has its own average dwell time to make the individual properties of each subsystem unneglected, which is more applicable and less conservative compared with ADT technique [9].

In fact, many studies focus on the asymptotic stability in the area of positive switched systems, which reflects the asymptotic behavior of the system in an infinite time interval. However, in many practical applications, one is more interested in what happens over a finite-time interval. Peter [10] has firstly defined finite-time stability (FTS) for linear deterministic systems, which means that, given a bound on the initial condition, the system state does not exceed a certain threshold during a specified time interval. Then Amato et al. [11] extended this definition to finite-time boundedness (FTB) when they dealt with the behavior of the state in the presence of external disturbances. Then many related results have been presented [12-17]. Recently, [18] first extends the concept of FTS to positive switched systems and gives some FTS conditions of positive switched systems. In [19], the problem of finite-time $L_{1}$ control for a class of positive switched linear systems with time-varying delays is considered and the concept of finite-time $L_{1}$ boundedness is also proposed. In [20], the problem of output feedback $L_{1}$ finite-time control for switched positive systems with timevarying delays is investigated via MDADT approach. On the other hand, when controlling a real plant, it is also desirable to design a control system which not only is stable but also guarantees an adequate level of performance. One approach to this problem is the guaranteed cost control. It has the advantage of providing an upper bound on a given system performance index and thus the system performance degradation incurred by the uncertainties or time delays is guaranteed to be less than this bound [21]. So it is necessary to study the design problem of guaranteed cost finite-time controller. There are some results about this problem; see [22-29] and references therein. These results mainly focus on nonpositive systems, involved in fuzzy systems [22, 27], continuous-time nonlinear systems [23], interconnected systems [24], Markov switching systems [25], stochastic systems [26, 28], neural networks [29], and so on. However, to the best of our knowledge, there are few results available on guaranteed cost finite-time control 
for positive switched linear systems with time-varying delays, which motivates our present study.

In this paper, the problem of the finite-time boundedness with guaranteed cost control for positive switched linear systems with time-varying delays is considered. Firstly, the definition of guaranteed cost finite-time boundedness is given, which is extended to positive switched linear systems with time-varying delays for the first time. Secondly, by using the MDADT approach and copositive Lyapunov-Krasovskii functional method, a static output feedback and a state feedback are designed, respectively, and sufficient conditions are obtained to guarantee that the closed-loop system is guaranteed cost finite-time boundedness (GCFTB). Such conditions can be easily solved by linear programming.

Notations. Throughout this paper, $A \succeq 0(>0, \preceq 0, \prec 0)$ means that all entries of matrix $A$ are nonnegative (positive, nonpositive, negative). $R_{+}^{n}$ is the $n$-dimensional nonnegative (positive) vector space, the $n$-dimensional Euclidean space is denoted by $R^{n}$ and $R^{n \times n}$ represents the space of $n \times n$ matrices with real entries, and $A^{T}$ is the transpose of a matrix $A$. $\|x\|=\sum_{k=1}^{n}\left|x_{k}\right|$, where $x_{k}$ is the $k$ th element of $x \in R^{n}$; if not explicitly stated, matrices are assumed to have compatible dimensions.

\section{Problem Statements and Preliminaries}

Consider the following positive switched linear systems with time-varying delays:

$$
\begin{aligned}
\dot{x}(t)= & A_{\sigma(t)} x(t)+A_{d \sigma(t)} x(t-d(t))+G_{\sigma(t)} u(t) \\
& +B_{\sigma(t)} w(t), \\
x(\theta)= & \varphi(\theta), \quad \theta \in[-\tau, 0], \\
y(t)= & C_{\sigma(t)} x(t),
\end{aligned}
$$

where $x(t) \in R^{n}, u(t) \in R^{m}$, and $y(t) \in R^{z}$ represent the system state, control input, and control output, respectively. $w(t) \in R^{l}$ is the disturbance input, which satisfies

$$
\exists d>0: \int_{0}^{T_{f}}\|w(t)\| d t<d .
$$

$\sigma(t):[0, \infty) \rightarrow \underline{S}=\{1,2, \ldots, S\}$ is the switching signal, where $S$ is the number of subsystems; $\forall p \in \underline{S}, A_{p}, A_{d p}, B_{p}, C_{p}$, and $G_{p}$ are constant matrices with appropriate dimensions, $p$ denotes $p$ th subsystems, and $t_{q}$ denotes the $q$ th switching instant; $\varphi(\theta)$ is the initial condition on $[-\tau, 0], \tau>0$, and $d(t)$ denotes the time-varying delay satisfying $0 \leq d(t) \leq$ $\tau, \dot{d}(t) \leq h<1$, where $\tau$ and $h$ are known positive constants.

Then we will present some definitions and lemmas for the following positive switched systems:

$$
\begin{aligned}
& \dot{x}(t)=A_{\sigma(t)} x(t)+A_{d \sigma(t)} x(t-d(t))+B_{\sigma(t)} w(t), \\
& x(\theta)=\varphi(\theta), \quad \theta \in[-\tau, 0], \\
& y(t)=C_{\sigma(t)} x(t) .
\end{aligned}
$$

The following definitions and lemmas are necessary for our further study.

Definition 1 (see [19]). System (3) is said to be positive if, for any initial conditions $\varphi(\theta) \succeq 0, \theta \in[-\tau, 0]$, disturbance input $w(t) \geq 0$, and any switching signals $\sigma(t)$, the corresponding trajectory $x(t) \geq 0$ holds for all $t \geq 0$.

Definition 2 (see [19]). A is called a Metzler matrix if the offdiagonal entries of matrix $A$ are nonnegative.

Lemma 3 (see [6]). A matrix $A$ is a Metzler matrix if and only if there exists a positive constant $\eta$ such that $A+\eta I_{n} \geq 0$.

Lemma 4 (see [19]). System (3) is positive if and only if $A_{p}, \forall p \in \underline{S}$, are Metzler matrices and $\forall p \in \underline{S}, A_{d p} \geq 0, B_{p} \geq$ $0, G_{p} \geq 0$, and $C_{p} \geq 0$.

Definition 5 (see [20]). For any switching signal $\sigma(t)$ and any $t_{2} \geq t_{1} \geq 0$, let $N_{\sigma p}\left(t_{1}, t_{2}\right)$ denote the switching numbers that the $p$ th subsystem is activated over the interval $\left[t_{1}, t_{2}\right)$ and $T_{p}\left(t_{1}, t_{2}\right)$ denote the total running time of the $p$ th subsystem over the interval $\left[t_{1}, t_{2}\right)$. If there exist $N_{0 p} \geq 0, \tau_{\alpha p}>0, \forall p \in$ $\underline{S}$, such that

$$
N_{\sigma p}\left(t_{1}, t_{2}\right) \leq N_{0 p}+\frac{T_{p}\left(t_{1}, t_{2}\right)}{\tau_{\alpha p}}, \quad \forall t_{2} \geq t_{1} \geq 0 .
$$

Then $\tau_{\alpha p}$ and $N_{0 p}$ are called MDADT and mode-dependent chatter bounds, respectively. Generally, we choose $N_{0 p}=0$.

Definition 6 (FTS). For a given time constant $T_{f}$ and vectors $\delta>\varepsilon>0$, positive switched system (3) with $w(t) \equiv 0$ is said to be finite-time stable with respect to $\left(\delta, \varepsilon, T_{f}, \sigma(t)\right)$, if

$$
\begin{aligned}
\sup _{-\tau \leq t \leq 0}\left\{x^{T}(t) \delta\right\} \leq 1 \Longrightarrow & \\
x^{T}(t) \varepsilon<1, & \\
& \forall t \in\left[0, T_{f}\right] .
\end{aligned}
$$

If the above condition is satisfied for any switching signals $\sigma(t)$, then system (3) is said to be uniformly finite-time stable with respect to $\left(\delta, \varepsilon, T_{f}\right)$.

Definition 7 (FTB). For a given time constant $T_{f}$ and vectors $\delta>\varepsilon>0$, positive switched system (3) is said to be finitetime boundedness with respect to $\left(\delta, \varepsilon, T_{f}, d, \sigma(t)\right)$, where $w(t)$ satisfies (2), if (5) applies.

Now we give some definitions about GCFTB for the positive switched systems (1).

Definition 8. Define the cost function of positive switched system (1) as follows:

$$
J=\int_{0}^{T_{f}}\left[x^{T}(t) R_{1}+u^{T}(t) R_{2}\right] d t
$$

where $R_{1}>0$ and $R_{2}>0$ are two given vectors. 
Remark 9. It should be noted that the proposed cost function is different from the existing literatures [21-24]; it is for the first time introduced in positive switched systems. This definition provides a more useful description, because it takes full advantage of the characteristics of nonnegative states of positive switched systems.

Definition 10 (GCFTB). For a given time constant $T_{f}$ and vectors $\delta>\varepsilon>0$, consider positive switched system (1) and cost function (6); if there exist a feedback control law $u(t)$ and a positive scalar $J^{*}$ such that the closed-loop system is FTB with respect to $\left(\delta, \varepsilon, T_{f}, d, \sigma(t)\right)$ and the cost function satisfies $J \leq J^{*}$, then the closed-loop system is called GCFTB, where $J^{*}$ is a guaranteed cost value and $u(t)$ is a guaranteed cost finite-time controller.

The aim of this paper is to design a static output feedback controller $u(t)=K_{\sigma(t)} y(t)$, a state feedback controller $u(t)=$ $\bar{K}_{\sigma(t)} x(t)$, and a class of switching signals $\sigma(t)$ for positive switched system (1) such that the corresponding closed-loop system is GCFTB.

\section{Main Results}

3.1. GCFTB Analysis. In this subsection, we will focus on the problem of GCFTB for positive switched system (3). The following theorem gives sufficient conditions of GCFTB for system (3) with the MDADT.

Theorem 11. Consider the positive switched system (3). For given constants $T_{f}, \lambda_{p}, \gamma$ and vectors $\delta>\varepsilon>0$ and $R_{1}>0$, if there exist a set of positive vectors $v_{p}, v_{p}, \vartheta_{p}, p \in \underline{S}$, and positive constants $\xi_{1}, \xi_{2}, \xi_{3}$, and $\xi_{4}$, the following inequalities hold:

$$
\begin{aligned}
& \Psi_{p}=\operatorname{diag}\left\{\psi_{p 1}, \psi_{p 2}, \ldots, \psi_{p n}, \psi_{p 1}^{\prime}, \psi_{p 2}^{\prime}, \ldots, \psi_{p n}^{\prime}\right\} \\
& \quad \leq 0, \\
& \xi_{1} \varepsilon \prec \nu_{p} \prec \xi_{2} \delta, \\
& v_{p} \prec \xi_{3} \delta, \\
& \vartheta_{p} \prec \xi_{4} \delta, \\
& \xi_{2} b_{p r}^{T} \delta<\gamma, \\
& \xi_{2}+\tau e^{\lambda \tau} \xi_{3}+\tau^{2} e^{\lambda \tau} \xi_{4}+\gamma d<\xi_{1} e^{-\kappa T_{f},}
\end{aligned}
$$

where

$$
\begin{aligned}
& \psi_{p r}=a_{p r}^{T}-\lambda_{p} v_{p r}+v_{p r}+\tau \vartheta_{p r}+R_{1}, \\
& \psi_{p r}^{\prime}=a_{d p r}^{T} v_{p}-(1-h) v_{p r} \\
& \quad \lambda=\max _{p \in \underline{S}}\left\{\lambda_{p}\right\}, r \in \underline{n}=\{1,2, \ldots, n\} .
\end{aligned}
$$

$a_{p r}\left(a_{d p r}\right)$ represents the rth column vector of the matrix $A_{p}\left(A_{d p}\right), v_{p}=\left[v_{p 1}, v_{p 2}, \ldots, v_{p n}\right]^{T}, v_{p}=\left[v_{p 1}, v_{p 2}, \ldots, v_{p n}\right]^{T}$, and $\vartheta_{p}=\left[\vartheta_{p 1}, \vartheta_{p 2}, \ldots, \vartheta_{p n}\right]^{T}$, and $v_{p r}, v_{p r}$, and $\vartheta_{p r}$ represent the rth elements of the vectors $v_{p}, v_{p}$, and $\vartheta_{p}$, respectively; then under the following MDADT scheme

$$
\begin{aligned}
\tau_{\alpha p}>\tau_{\alpha p}{ }^{*} & \\
& =\max \left\{\frac{T_{f} \ln \mu_{p}}{\ln \left(\xi_{1} e^{-\lambda_{p} T_{f}}\right)-\ln \left(\xi_{2}+\tau e^{\lambda_{p} \tau} \xi_{3}+\tau^{2} e^{\lambda_{p} \tau} \xi_{4}+\gamma d\right)},\right. \\
& \left.\frac{\ln \mu_{p}}{\lambda}\right\},
\end{aligned}
$$

system (3) is GCFTB with respect to $\left(\delta, \varepsilon, T_{f}, d, \sigma(t)\right)$, where $\mu_{p}\left(\mu_{p} \geq 1\right)$ satisfies

$$
\begin{aligned}
& v_{p} \preceq \mu_{p} v_{q}, \\
& v_{p} \preceq \mu_{p} v_{q}, \\
& \vartheta_{p} \preceq \mu_{p} \vartheta_{q},
\end{aligned}
$$

\section{$\forall p, q \in \underline{S}$.}

And the guaranteed cost value of system (3) is given by

$$
\begin{aligned}
J & =\int_{0}^{T_{f}} x^{T}(s) R_{1} d s \leq J^{*} \\
& =e^{2 \lambda T_{f}}\left(\xi_{2}+\tau e^{\lambda \tau} \xi_{3}+\tau^{2} e^{\lambda \tau} \xi_{4}+\gamma d\right) .
\end{aligned}
$$

Proof. Construct the following copositive LyapunovKrasovskii functional for system (3):

$$
\begin{aligned}
V_{\sigma(t)}(t, x(t))= & x^{T}(t) v_{p}+\int_{t-d(t)}^{t} e^{\lambda_{p}(t-s)} x^{T}(s) v_{p} d s \\
& +\int_{-\tau}^{0} \int_{t+\theta}^{t} e^{\lambda_{p}(t-s)} x^{T}(s) \vartheta_{p} d s d \theta,
\end{aligned}
$$

where $v_{p}, v_{p}$, and $\vartheta_{p} \in R_{+}^{n}, \forall p \in \underline{S}$.

For the sake of simplicity, $V_{\sigma(t)}(t, x(t))$ is written as $V_{\sigma(t)}(t)$ in this paper.

Along the trajectory of system (3), we have

$$
\begin{aligned}
\dot{V}_{\sigma(t)}(t)= & x^{T}(t) A_{p}^{T} v_{p}+x^{T}(t-d(t)) A_{d p}^{T} v_{p} \\
& +w^{T}(t) B_{p}^{T} v_{p} \\
& +\lambda_{p} \int_{t-d(t)}^{t} e^{\lambda_{p}(t-s)} x^{T}(s) v_{p} d s+x^{T}(s) v_{p} \\
& -(1-\dot{d}(t)) e^{\lambda_{p} d(t)} x^{T}(t-d(t)) v_{p} \\
& +\lambda_{p} \int_{-\tau}^{0} \int_{t+\theta}^{t} e^{\lambda_{p}(t-s)} x^{T}(s) \vartheta_{p} d s d \theta \\
& +\tau x^{T}(t) \vartheta_{p}-\int_{-\tau}^{0} e^{-\lambda_{p} \theta} x^{T}(t+\theta) \vartheta_{p} d \theta
\end{aligned}
$$




$$
\begin{aligned}
\leq & x^{T}(t) A_{p}^{T} v_{p}+x^{T}(t-d(t)) A_{d p}^{T} \nu_{p} \\
& +w^{T}(t) B_{p}^{T} v_{p} \\
& +\lambda_{p} \int_{t-d(t)}^{t} e^{\lambda_{p}(t-s)} x^{T}(s) v_{p} d s+x^{T}(s) v_{p} \\
& -(1-h) x^{T}(t-d(t)) v_{p} \\
& +\lambda_{p} \int_{-\tau}^{0} \int_{t+\theta}^{t} e^{\lambda_{p}(t-s)} x^{T}(s) \vartheta_{p} d s d \theta \\
& +\tau x^{T}(t) \vartheta_{p}-\int_{t-d(t)}^{t} x^{T}(s) \vartheta_{p} d s .
\end{aligned}
$$

Combining (8), (9), (15), and (16) leads to

$$
\begin{aligned}
\dot{V}_{\sigma(t)}( & (t)-\lambda_{p} V_{\sigma(t)}(t)+x^{T}(t) R_{1} \\
= & x^{T}(t) A_{p}^{T} v_{p}-\lambda_{p} x^{T}(t) v_{p}+x^{T}(s) v_{p} \\
& +\tau x^{T}(t) \vartheta_{p}+x^{T}(t-d(t)) A_{d p}^{T} v_{p} \\
& -(1-h) x^{T}(t-d(t)) v_{p}+w^{T}(t) \xi_{2} B_{p}^{T} \delta \\
& -\int_{t-d(t)}^{t} x^{T}(s) \vartheta_{p} d s+x^{T}(t) R_{1} \\
\leq & x^{T}(t)\left(A_{p}^{T} v_{p}-\lambda_{p} v_{p}+v_{p}+\tau \vartheta_{p}+R_{1}\right) \\
& +x^{T}(t-d(t))\left(A_{d p}^{T} v_{p}-(1-h) v_{p}\right) \\
& +\gamma\|w(t)\| .
\end{aligned}
$$

Substituting (7) into (17) yields

$$
\dot{V}_{\sigma(t)}(t)-\lambda_{p} V_{\sigma(t)}(t)+x^{T}(t) R_{1}-\gamma\|w(t)\| \leq 0 .
$$

It implies

$$
\dot{V}_{\sigma(t)}(t)-\lambda_{p} V_{\sigma(t)}(t)-\gamma\|w(t)\| \leq 0
$$

Integrating both sides of (19) during the period $\left[t_{k}, t\right)$ for $t \in$ $\left[t_{k}, t_{k+1}\right)$ leads to

$$
\begin{aligned}
V_{\sigma(t)}(t) \leq & e^{\lambda_{\sigma\left(t_{k}\right)}\left(t-t_{k}\right)} V_{\sigma\left(t_{k}\right)}\left(t_{k}\right) \\
& +\gamma \int_{t_{k}}^{t} e^{\lambda_{\sigma\left(t_{k}\right)}(t-s)} w^{T}(s) d s
\end{aligned}
$$

For any $T_{f}>0$, let $N$ be the switching number of $\sigma(t)$ over $\left[0, T_{f}\right)$, and denote $t_{1}, t_{2}, \ldots, t_{k}, \ldots, t_{N}$ as the switching instants over the interval $\left[0, T_{f}\right)$. Then, for $t \in$ $\left[0, T_{f}\right), V_{\sigma\left(t_{k}\right)}\left(t_{k}\right) \leq \mu_{\sigma\left(t_{k}\right)} V_{\sigma\left(t_{k}-\right)}\left(t_{k}{ }^{-}\right)$is easily obtained from (13) and (15). Combining with (19), we have

$$
\begin{aligned}
& V_{\sigma(t)}(t) \leq e^{\lambda_{\sigma\left(t_{k}\right)}\left(t-t_{k}\right)} V_{\sigma\left(t_{k}\right)}\left(t_{k}\right) \\
& +\gamma \int_{t_{k}}^{t} e^{\lambda_{\sigma\left(t_{k}\right)}(t-s)} w^{T}(s) d s \\
& \leq \mu_{\sigma\left(t_{k}\right)} e^{\lambda_{\sigma\left(t_{k}\right)}\left(t-t_{k}\right)} V_{\sigma\left(t_{k}^{-}\right)}\left(t_{k}^{-}\right) \\
& +\gamma \int_{t_{k}}^{t} e^{\lambda_{\sigma\left(t_{k}\right)}(t-s)} w^{T}(s) d s \\
& \leq \mu_{\sigma\left(t_{k}\right)} e^{\lambda_{\sigma\left(t_{k}\right)}\left(t-t_{k}\right)}\left[e^{\lambda_{\sigma\left(t_{k}-1\right)}\left(t_{k}-t_{k-1}\right)} V_{\sigma\left(t_{k-1}\right)}\left(t_{k-1}\right)\right. \\
& \left.+\gamma \int_{t_{k-1}}^{t_{k}} e^{\lambda_{\sigma\left(t_{k-1}\right)}\left(t_{k}-s\right)} w^{T}(s) d s\right] \\
& +\gamma \int_{t_{k}}^{t} e^{\lambda_{\sigma\left(t_{k}\right)}(t-s)} w^{T}(s) d s \leq \cdots \leq\left(\prod_{i=1}^{k} u_{\sigma\left(t_{i}\right)}\right) \\
& \cdot e^{\lambda_{\sigma\left(t_{k}\right)}\left(t-t_{k}\right)} e^{\sum_{i=1}^{k-1} \lambda_{\sigma\left(t_{i}\right)}\left(t_{i}-t_{i-1}\right)} V_{\sigma(0)}(0)+\left(\prod_{i=1}^{k} u_{\sigma\left(t_{i}\right)}\right) \\
& \cdot \gamma \int_{0}^{t_{1}} e^{\lambda_{\sigma(0)}\left(t_{1}-s\right)} w^{T}(s) d s+\left(\prod_{i=2}^{k} u_{\sigma\left(t_{i}\right)}\right) \\
& \cdot \gamma \int_{t_{1}}^{t_{2}} e^{\lambda_{\sigma\left(t_{1}\right)}\left(t_{2}-s\right)} w^{T}(s) d s+\cdots \\
& +\gamma \int_{t_{k}}^{t} e^{\lambda_{\sigma\left(t_{k}\right)}(t-s)} w^{T}(s) d s \leq\left(\prod_{p=1}^{S} u_{p}^{N_{\sigma p}(0, t)}\right) \\
& \cdot e^{\sum_{p=1}^{S} \lambda_{p} T_{p}(0, t)} V_{\sigma(0)}(0) \\
& +\gamma \int_{0}^{t}\left(\prod_{p=1}^{S} u_{p}^{N_{\sigma p}(0, t)}\right) e^{\sum_{p=1}^{S} \lambda_{p} T_{p}(s, t)} w^{T}(s) d s .
\end{aligned}
$$

According to the definition of MDADT, one can obtain from (21)

$$
\begin{aligned}
& V_{\sigma(t)}(t) \\
& \quad \leq\left(\prod_{p=1}^{S} u_{p}^{T_{p}(0, t) / \tau_{\alpha p}}\right) e^{\sum_{p=1}^{S} \lambda_{p} T_{p}(0, t)}\left(V_{\sigma(0)}(0)+\gamma d\right) \\
& \quad=e^{\sum_{p=1}^{S}\left(\lambda_{p}+\ln \mu_{p} / \tau_{\alpha p}\right) T_{p}(0, t)}\left(V_{\sigma(0)}(0)+\gamma d\right) .
\end{aligned}
$$

Choose $\kappa=\max _{p \in \underline{S}}\left(\lambda_{p}+\ln \mu_{p} / \tau_{\alpha p}\right)$. Therefore, for $t \in\left[0, T_{f}\right)$

$$
V_{\sigma(t)}(t) \leq e^{\kappa T_{f}}\left(V_{\sigma(0)}(0)+\gamma d\right)
$$

Noting the definition of $V_{\sigma(t)}(t)$ and (8), we have

$$
V_{\sigma(t)}(t) \geq \xi_{1} x^{T}(t) \varepsilon,
$$




$$
\begin{aligned}
V_{\sigma(0)}(0) \leq & \xi_{2} x^{T}(0) \delta+\tau e^{\lambda \tau} \xi_{3} \sup _{-\tau \leq \theta \leq 0}\left\{x^{T}(\theta) \delta\right\} \\
& +\tau^{2} e^{\lambda \tau} \xi_{4} \sup _{-\tau \leq \theta \leq 0}\left\{x^{T}(\theta) \delta\right\} \\
\leq & \left(\xi_{2}+\tau e^{\lambda \tau} \xi_{3}+\tau^{2} e^{\lambda \tau} \xi_{4}\right) \sup _{-\tau \leq \theta \leq 0}\left\{x^{T}(\theta) \delta\right\} \\
\leq & \xi_{2}+\tau e^{\lambda \tau} \xi_{3}+\tau^{2} e^{\lambda \tau} \xi_{4} .
\end{aligned}
$$

Combining (21)-(25), we obtain

$$
x^{T}(t) \varepsilon \leq \frac{1}{\xi_{1}} e^{\kappa T_{f}}\left(\xi_{2}+\tau e^{\lambda \tau} \xi_{3}+\tau^{2} e^{\lambda \tau} \xi_{4}+\gamma d\right) .
$$

Substituting (10) into (26), one has

$$
x^{T}(t) \varepsilon<1 .
$$

From Definition 7, we conclude that system (3) is FTB with respect to $\left(\delta, \varepsilon, T_{f}, d, \sigma(t)\right)$.

Next, we will give the guaranteed cost value of system (3).

According to (17), denoting $\nabla(t)=\gamma\|w(t)\|-x^{T}(t) R_{1}$ and integrating both sides of (18) from $t_{k}$ to $t$ for $t \in\left[t_{k}, t_{k+1}\right]$, it gives rise to

$$
V_{\sigma(t)}(t) \leq e^{\lambda_{p}\left(t-t_{k}\right)} V_{\sigma\left(t_{k}\right)}\left(t_{k}\right)+\int_{t_{k}}^{t} e^{\lambda_{p}(t-s)} \nabla(s) d s .
$$

Similar to the proof process of (21), for any $t \in\left[0, T_{f}\right]$, we can obtain

$$
\begin{aligned}
V_{\sigma(t)}(t) \leq & \left(\prod_{p=1}^{S} u_{p}^{N_{\sigma p}(0, t)}\right) e^{\sum_{p=1}^{S} \lambda T_{p}(0, t)} V_{\sigma(0)}(0) \\
& +\int_{0}^{t}\left(\prod_{p=1}^{S} u_{p}^{N_{\sigma p}(s, t)}\right) e^{\sum_{p=1}^{S} \lambda T_{p}(s, t)} \nabla(s) d s,
\end{aligned}
$$

where $\lambda=\max _{i \in \underline{s}} \lambda_{p}$. From (29), we can get

$$
\begin{aligned}
& \int_{0}^{t}\left(\prod_{p=1}^{S} u_{p}^{N_{\sigma p}(s, t)}\right) e^{\sum_{p=1}^{S} \lambda T_{p}(s, t)} x^{T}(s) R_{1} d s \\
& \leq\left(\prod_{p=1}^{S} u_{p}^{N_{\sigma p}(0, t)}\right) e^{\sum_{p=1}^{S} \lambda T_{p}(0, t)} V_{\sigma(0)}(0) \\
& \quad+\gamma \int_{0}^{t}\left(\prod_{p=1}^{S} u_{p}^{N_{\sigma p}(s, t)}\right) e^{\sum_{p=1}^{S} \lambda T_{p}(s, t)}\|w(s)\| d s .
\end{aligned}
$$

Multiplying both sides of (30) by $\prod_{p=1}^{S} \mu^{-N_{\sigma p}(0, t)}$ leads to

$$
\begin{aligned}
\int_{0}^{t}( & \left.\prod_{p=1}^{s} u_{p}^{-N_{\sigma p}(0, s)}\right) e^{\lambda(t-s)} x^{T}(s) R_{1} d s \\
\leq & e^{\lambda T_{f}} V_{\sigma(0)}(0) \\
& +\gamma \int_{0}^{t}\left(\prod_{p=1}^{s} u_{p}^{-N_{\sigma p}(0, s)}\right) e^{\lambda(t-s)}\|w(s)\| d s .
\end{aligned}
$$

Noting that $N_{\sigma p}(0, s) \leq T_{p}(0, s) / \tau_{\alpha p}$ and $\tau_{\alpha p}>\ln \mu_{p} / \lambda$, we obtain that $0<N_{\sigma p}(0, s) \leq T_{p}(0, s) / \tau_{\alpha p} \leq \lambda s / \ln \mu_{p}$; that is, $e^{-\lambda s} \leq \mu_{p}{ }^{-N_{p}(0, s)}<1$.

Then (31) can be turned into

$$
\begin{aligned}
& \int_{0}^{t} e^{-\lambda s} e^{\lambda(t-s)} x^{T}(s) R_{1} d s \\
& \quad \leq \int_{0}^{t} \mu^{-N_{\sigma p}(0, s)} e^{\lambda(t-s)} x^{T}(s) R_{1} d s \\
& \leq e^{\lambda T_{f}} V_{\sigma(0)}(0)+\gamma \int_{0}^{t} e^{\lambda(t-s)}\|w(s)\| d s .
\end{aligned}
$$

Let $t=T_{f}$; then multiplying both sides of (32) by $e^{-\lambda T_{f}}$ leads to

$$
\begin{aligned}
\int_{0}^{T_{f}} e^{-2 \lambda s} x^{T}(s) R_{1} d s \leq & V_{\sigma(0)}(0) \\
& \quad+\gamma \int_{0}^{T_{f}} e^{-\lambda s}\|w(s)\| d s \\
\leq & V_{\sigma(0)}(0)+\gamma \int_{0}^{T_{f}}\|w(s)\| d s .
\end{aligned}
$$

Substituting (2) into (33) yields

$$
e^{-2 \lambda T_{f}} \int_{0}^{T_{f}} x^{T}(s) R_{1} d s \leq V_{\sigma(0)}(0)+\gamma d
$$

which can be rewritten as

$$
\int_{0}^{T_{f}} x^{T}(s) R_{1} d s \leq e^{2 \lambda T_{f}}\left(V_{\sigma(0)}(0)+\gamma d\right) .
$$

Substituting (25) into (35), the guaranteed cost value of system (3) is given by

$$
\begin{aligned}
J & =\int_{0}^{T_{f}} x^{T}(s) R_{1} d s \leq J^{*} \\
& =e^{2 \lambda T_{f}}\left(\xi_{2}+\tau e^{\lambda \tau} \xi_{3}+\tau^{2} e^{\lambda \tau} \xi_{4}+\gamma d\right) .
\end{aligned}
$$

Therefore, according to Definition 10, we can conclude that system (3) is GCFTB with respect to $\left(\delta, \varepsilon, T_{f}, d, \sigma(t)\right)$. Thus, the proof is completed.

Remark 12. In Theorem 11, if $\mu_{p} \equiv 1$ in (12), then one can obtain $\tau_{\alpha p}{ }^{*}=0$, which means that the switching signal can be arbitrary.

When the exogenous disturbance $w(t)=0$, the result on guaranteed cost finite-time stability can be obtained as follows.

Corollary 13. Consider switched positive system (3) with $w(t)=0$. For given constants $T_{f}, \lambda$, and vectors $\delta>\varepsilon>$ $0, R_{1}>0$, if there exist a set of positive vectors $v_{p}, v_{p}, \vartheta_{p}, p \in$ $\underline{S}$, and positive constants $\xi_{1}, \xi_{2}, \xi_{3}$, and $\xi_{4}$, such that (7), (8), and the following inequality holds

$$
\xi_{2}+\tau e^{\lambda \tau} \xi_{3}+\tau^{2} e^{\lambda \tau} \xi_{4}<\xi_{1} e^{-\kappa T_{f}}
$$


then under the following MDADT scheme

$$
\begin{aligned}
\tau_{a p} & >\tau_{a p}{ }^{*} \\
& =\frac{T_{f} \ln \mu_{p}}{\ln \left(\xi_{1} e^{-\lambda_{p} T_{f}}\right)-\ln \left(\xi_{2}+\tau e^{\lambda \tau} \xi_{3}+\tau^{2} e^{\lambda \tau} \xi_{4}\right)}
\end{aligned}
$$

system (3) is guaranteed cost finite-time stable to $\left(\delta, \varepsilon, T_{f}, d, \sigma(t)\right)$, where $\mu_{p} \geq 1$ satisfies (13); the corresponding guaranteed cost value is given by

$$
J^{*}=e^{2 \lambda T_{f}}\left(\xi_{2}+\tau e^{\lambda \tau} \xi_{3}+\tau^{2} e^{\lambda \tau} \xi_{4}\right) .
$$

3.2. GCFTB Controller Design. In this section, we are concerned with the GCFTB controller design of positive switched system (1). The static output feedback control law and state feedback control law will be designed to ensure the close-loop system (1) is GCFTB, respectively.

3.2.1. Static Output Feedback Controller Design. Consider system (1); under the controller $u(t)=K_{\sigma(t)} y(t)$, the corresponding closed-loop system is given by

$$
\begin{aligned}
\dot{x}(t)= & \left(A_{\sigma(t)}+G_{\sigma(t)} K_{\sigma(t)} C_{\sigma(t)}\right) x(t) \\
& +A_{d \sigma(t)} x(t-d(t))+B_{\sigma(t)} w(t), \\
x(\theta)= & \varphi(\theta), \quad \theta \in[-\tau, 0], \\
y(t)= & C_{\sigma(t)} x(t) .
\end{aligned}
$$

By Lemma 3, to guarantee the positivity of system (40), $A_{p}+G_{p} K_{p} C_{p}$ should be Metzler matrices, $\forall p \in \underline{S}$. Theorem 14 gives some sufficient conditions to guarantee that the closedloop system (1) is GCFTB.

Theorem 14. Consider the positive switched system (1). For given constants $T_{f}, \lambda_{p}, \gamma$ and vectors $\delta>\varepsilon>0, R_{1}>0$, and $R_{2}>0$, if there exist a set of positive vectors $v_{p}, v_{p}, \vartheta_{p}, p \in$ $\underline{S}$, and positive constants $\xi_{1}, \xi_{2}, \xi_{3}$, and $\xi_{4},(8)-(10)$ and the following conditions hold:

$$
\begin{aligned}
& A_{p}+G_{p} K_{p} C_{p} \text { are Metzler matrices, } \\
& \bar{\Psi}_{p}=\operatorname{diag}\left\{\bar{\psi}_{p 1}, \bar{\psi}_{p 2}, \ldots, \bar{\psi}_{p n}, \bar{\psi}_{p 1}^{\prime}, \bar{\psi}_{p 2}^{\prime}, \ldots, \bar{\psi}_{p n}^{\prime}\right\} \\
& \quad \leq 0,
\end{aligned}
$$

where

$$
\begin{aligned}
& \bar{\psi}_{p r}=a_{p r}^{T}-\lambda_{p} v_{p r}+v_{p r}+\tau \vartheta_{p r}+R_{1}+g_{p r}, \\
& \bar{\psi}_{p r}^{\prime}=a_{d p r}^{T} v_{p}-(1-h) v_{p r} \\
& \quad \lambda=\max _{p \in \underline{S}}\left\{\lambda_{p}\right\}, r \in \underline{n}=\{1,2, \ldots, n\} .
\end{aligned}
$$

$g_{p}=C_{p}^{T} K_{p}^{T}\left(G_{p}^{T} \nu_{p}+R_{2}\right)$, where $g_{p r}$ represents the rth elements of vector $g_{p}, a_{p r}\left(a_{d p r}\right)$ represents the rth column vector of the matrix $A_{p}\left(A_{d p}\right)$, and $v_{p}=\left[v_{p 1}, v_{p 2}, \ldots, v_{p n}\right]^{T}, v_{p}=$ $\left[v_{p 1}, v_{p 2}, \ldots, v_{p n}\right]^{T}$, and $\vartheta_{p}=\left[\vartheta_{p 1}, \vartheta_{p 2}, \ldots, \vartheta_{p n}\right]^{T}, v_{p r}, v_{p r}$, and $\vartheta_{p r}$ represent the rth elements of the vectors $v_{p}, v_{p}$, and $\vartheta_{p}$, respectively, and $\mu_{p}\left(\mu_{p} \geq 1\right)$ satisfies (13); then under the MDADT scheme (12), the resulting closed-loop system (40) is $G C F T B$ with respect to $\left(\delta, \varepsilon, T_{f}, d, \sigma(t)\right)$ and the guaranteed cost value of system (40) is given by

$$
\begin{aligned}
J & =\int_{0}^{T_{f}}\left(x^{T}(s) R_{1}+x^{T}(s) C_{\sigma(t)}^{T} K_{\sigma(t)}^{T} R_{2}\right) d s \leq J^{*} \\
& =e^{2 \lambda T_{f}}\left(\xi_{2}+\tau e^{\lambda \tau} \xi_{3}+\tau^{2} e^{\lambda \tau} \xi_{4}+\gamma d\right) .
\end{aligned}
$$

Proof. By Lemma 3, we know that $A_{p}+G_{p} K_{p} C_{p}$ is a Metzler matrix for each $p \in \underline{S}$. According to Lemma 4, system (40) is positive if $A_{d p}, B_{p}, G_{p}$, and $C_{p}, p \in \underline{S}$ are all nonnegative. Replacing $A_{p}$ in (7) with $A_{p}+G_{p} K_{p} C_{p}$ and letting $g_{p}=$ $C_{p}^{T} K_{p}^{T}\left(G_{p}^{T} \nu_{p}+R_{2}\right)$, similar to Theorem 11 , we easily obtain that the resulting closed-loop system (40) is GCFTB with respect to $\left(\delta, \varepsilon, T_{f}, d, \sigma(t)\right)$ and the guaranteed cost value is given by $(44)$.

The proof is completed.

3.2.2. State Feedback Controller Design. Consider system (1); under the controller $u(t)=\bar{K}_{\sigma(t)} x(t)$, the corresponding closed-loop system is given by

$$
\begin{aligned}
\dot{x}(t)= & \left(A_{\sigma(t)}+G_{\sigma(t)} \bar{K}_{\sigma(t)}\right) x(t)+A_{d \sigma(t)} x(t-d(t)) \\
& +B_{\sigma(t)} w(t) \\
x(\theta)= & \varphi(\theta), \quad \theta \in[-\tau, 0], \\
y(t)= & C_{\sigma(t)} x(t) .
\end{aligned}
$$

By Lemma 3, to guarantee the positivity of system (45), $A_{p}+G_{p} \bar{K}_{p}$ should be Metzler matrices, $\forall p \in \underline{S}$. The following Theorem 15 gives some sufficient conditions to guarantee that closed-loop system (1) is GCFTB.

Theorem 15. Consider the positive switched system (1). For given constants $T_{f}, \lambda_{p}, \gamma$ and vectors $\delta>\varepsilon>0, R_{1}>0$, and $R_{2}>0$, if there exist a set of positive vectors $v_{p}, v_{p}, \vartheta_{p}, p \in$ $\underline{S}$, and positive constants $\xi_{1}, \xi_{2}, \xi_{3}$, and $\xi_{4},(8)-(10)$ and the following conditions hold:

$$
\begin{aligned}
& A_{p}+G_{p} \bar{K}_{p} \text { are Metzler matrices, } \\
& \bar{\Psi}_{p}=\operatorname{diag}\left\{\bar{\psi}_{p 1}, \bar{\psi}_{p 2}, \ldots, \bar{\psi}_{p n}, \bar{\psi}_{p 1}^{\prime}, \bar{\psi}_{p 2}^{\prime}, \ldots, \bar{\psi}_{p n}^{\prime}\right\} \\
& \quad \leq 0
\end{aligned}
$$

where

$$
\begin{aligned}
& \bar{\psi}_{p r}=a_{p r}^{T}-\lambda_{p} v_{p r}+v_{p r}+\tau \vartheta_{p r}+R_{1}+g_{p r}, \\
& \bar{\psi}_{p r}^{\prime}=a_{d p r}^{T} \nu_{p}-(1-h) v_{p r}, \\
& \quad \lambda=\max _{p \in \underline{S}}\left\{\lambda_{p}\right\}, r \in \underline{n}=\{1,2, \ldots, n\} .
\end{aligned}
$$


$g_{p}=\bar{K}_{p}^{T}\left(G_{p}^{T} v_{p}+R_{2}\right)$, where $g_{p r}$ represents the rth elements of vector $g_{p}, a_{p r}\left(a_{d p r}\right)$ represents the rth column vector of the matrix $A_{p}\left(A_{d p}\right)$, and $v_{p}=\left[v_{p 1}, v_{p 2}, \ldots, v_{p n}\right]^{T}, v_{p}=$ $\left[v_{p 1}, v_{p 2}, \ldots, v_{p n}\right]^{T}$, and $\vartheta_{p}=\left[\vartheta_{p 1}, \vartheta_{p 2}, \ldots, \vartheta_{p n}\right]^{T}, v_{p r}, v_{p r}$, and $\vartheta_{p r}$ represent the rth elements of the vectors $v_{p}, v_{p}$, and $\vartheta_{p}$, respectively, and $\mu_{p}\left(\mu_{p} \geq 1\right)$ satisfies (13); then under the $M D A D T$ scheme (12), the resulting closed-loop system (45) is $G C F T B$ with respect to $\left(\delta, \varepsilon, T_{f}, d, \sigma(t)\right)$ and the guaranteed cost value of system (45) is given by

$$
\begin{aligned}
J & =\int_{0}^{T_{f}}\left(x^{T}(s) R_{1}+x^{T}(s) \bar{K}_{\sigma(s)}^{T} R_{2}\right) d s \leq J^{*} \\
& =e^{2 \lambda T_{f}}\left(\xi_{2}+\tau e^{\lambda \tau} \xi_{3}+\tau^{2} e^{\lambda \tau} \xi_{4}+\gamma d\right) .
\end{aligned}
$$

Proof. By Lemma 3, we know that $A_{p}+G_{p} \bar{K}_{p}$ is a Metzler matrix for each $p \in \underline{S}$. According to Lemma 4, system (45) is positive if $A_{d p}, B_{p}, G_{p}$, and $C_{p}$ are all nonnegative. Replacing $A_{p}$ in (7) with $A_{p}+G_{p} \bar{K}_{p}$ and letting $g_{p}=$ $\bar{K}_{p}^{T}\left(G_{p}^{T} v_{p}+R_{2}\right)$, similar to Theorem 11, we easily obtain that the resulting closed-loop system (45) is GCFTB with respect to $\left(\delta, \varepsilon, T_{f}, d, \sigma(t)\right)$ and the guaranteed cost value is given by (49).

The proof is completed.

Next, a procedure is presented to obtain the feedback gain matrices $K_{p}\left(\bar{K}_{p}\right), p \in \underline{S}$.

Step 1. By adjusting the parameters $\lambda_{p}$ and solving (8)-(10), (13), (42), and (47) via linear programming, positive vectors $v_{p}, v_{p}, \vartheta_{p}$, and $g_{p}$ can be obtained.

Step 2. Substituting $v_{p}$ and $g_{p}$ into $g_{p}=C_{p}^{T} K_{p}^{T}\left(G_{p}^{T} v_{p}+R_{2}\right)$ (or $\left.g_{p}=\bar{K}_{p}^{T}\left(G_{p}^{T} v_{p}+R_{2}\right)\right), K_{p}\left(\bar{K}_{p}\right)$ can be obtained.

Step 3. The gain $K_{p}\left(\bar{K}_{p}\right)$ is substituted into $A_{p}+G_{p} K_{p} C_{p}$ (or $\left.A_{p}+G_{p} \bar{K}_{p}\right)$. If $A_{p}+G_{p} K_{p} C_{p}\left(\right.$ or $\left.A_{p}+G_{p} \bar{K}_{p}\right)$ are Metzler matrices, then $K_{p}\left(\bar{K}_{p}\right)$ are admissible. Otherwise, return to Step 1.

\section{Numerical Example}

In this section, a numerical example in [6] is provided to show the effectiveness of the proposed approach. Consider positive switched system (1) with the parameters as follows:

$$
\begin{aligned}
A_{1} & =\left[\begin{array}{cc}
-3.5 & 0.1 \\
0.3 & -3
\end{array}\right], \\
A_{d 1} & =\left[\begin{array}{ll}
0.2 & 0.5 \\
0.8 & 0.6
\end{array}\right], \\
B_{1} & =\left[\begin{array}{ll}
0.2 & 0.1 \\
0.1 & 0.3
\end{array}\right],
\end{aligned}
$$

$$
\begin{aligned}
G_{1} & =\left[\begin{array}{l}
1 \\
2
\end{array}\right], \\
C_{1} & =\left[\begin{array}{ll}
0.1 & 0.2 \\
0.4 & 0.1
\end{array}\right], \\
A_{2} & =\left[\begin{array}{ll}
-5 & 0.1 \\
0.1 & -2.5
\end{array}\right], \\
A_{d 2} & =\left[\begin{array}{ll}
0.6 & 0.3 \\
0.8 & 0.5
\end{array}\right], \\
B_{2} & =\left[\begin{array}{ll}
0.3 & 0.1 \\
0.2 & 0.1
\end{array}\right], \\
G_{2} & =\left[\begin{array}{l}
2 \\
1
\end{array}\right], \\
C_{2} & =\left[\begin{array}{ll}
0.2 & 0.5 \\
0.3 & 0.1
\end{array}\right], \\
R_{1} & =\left[\begin{array}{l}
0.2 \\
0.3
\end{array}\right], \\
\mathcal{E} & =\left[\begin{array}{l}
0.1 \\
0.1
\end{array}\right], \\
\delta & =\left[\begin{array}{l}
1 \\
2
\end{array}\right] . \\
R_{2} & =\left[\begin{array}{l}
0.3 \\
0.2
\end{array}\right],
\end{aligned}
$$

Assuming that $d(t)=0.2+0.2 \sin (t)$, we can get $\tau=0.4$ and $h=0.2$.

Let

$$
\begin{aligned}
& w(t)=\left[\begin{array}{l}
f_{1}(t) \\
f_{2}(t)
\end{array}\right] \\
& f_{1}(t)=f_{2}(t)= \begin{cases}\frac{1+\sin (t)}{t} & 3 \leq t \leq 10, \\
0 & \text { others. }\end{cases}
\end{aligned}
$$

Choosing the parameters $T_{f}=10, \lambda_{1}=0.2, \lambda_{2}=0.14, \gamma=$ 1 , and $\mu_{1}=\mu_{2}=1.4$ and solving the inequalities in Theorem 14 by linear programming, we get

$$
\begin{aligned}
& v_{1}=\left[\begin{array}{l}
1.2590 \\
2.1813
\end{array}\right], \\
& v_{1}=\left[\begin{array}{l}
1.8096 \\
3.1278
\end{array}\right], \\
& \vartheta_{1}=\left[\begin{array}{l}
1.4848 \\
1.9703
\end{array}\right],
\end{aligned}
$$




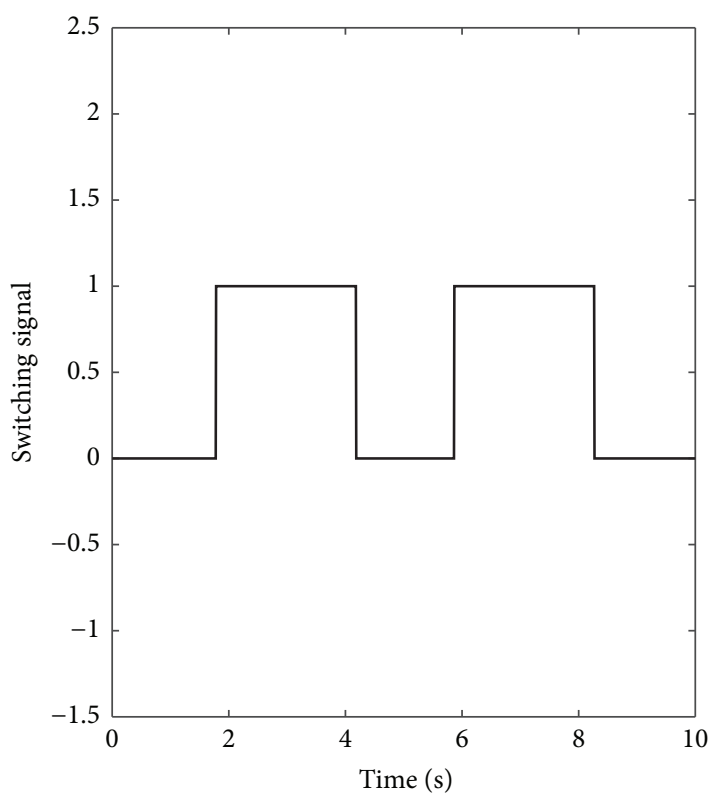

FIGURE 1: Switching signal of system (1).

$$
\begin{aligned}
& v_{2}=\left[\begin{array}{l}
1.1825 \\
2.2998
\end{array}\right], \\
& v_{2}=\left[\begin{array}{l}
1.8191 \\
3.0665
\end{array}\right], \\
& \vartheta_{2}=\left[\begin{array}{l}
1.4945 \\
1.9646
\end{array}\right], \\
& g_{1}=\left[\begin{array}{l}
0.7149 \\
0.7630
\end{array}\right], \\
& g_{2}=\left[\begin{array}{l}
0.8474 \\
0.7229
\end{array}\right] .
\end{aligned}
$$

(I) By $g_{p}=C_{p}^{T} K_{p}^{T}\left(G_{p}^{T} v_{p}+R_{2}\right)$, we obtain the static output feedback controller:

$$
\begin{aligned}
& K_{1}=\left[\begin{array}{ll}
0.1900 & 0.4910
\end{array}\right], \\
& K_{2}=\left[\begin{array}{ll}
0.2650 & 0.1050
\end{array}\right] .
\end{aligned}
$$

(II) By $g_{p}=\bar{K}_{p}^{T}\left(G_{p}^{T} v_{p}+R_{2}\right)$, we obtain the state feedback controller:

$$
\begin{aligned}
& \bar{K}_{1}=\left[\begin{array}{ll}
0.2881 & 0.1998
\end{array}\right], \\
& \bar{K}_{2}=\left[\begin{array}{ll}
0.1275 & 0.4291
\end{array}\right] .
\end{aligned}
$$

It is easy to verify that (41) and (46) are satisfied. Then, according to (12), we get $\tau_{\alpha 1}{ }^{*}=1.6824$ and $\tau_{\alpha 2}{ }^{*}=2.4034$.

The simulation results are shown in Figures 1-5; the initial conditions of system (1) are $x(0)=\left[\begin{array}{ll}0.5 & 0.2\end{array}\right]^{T}$

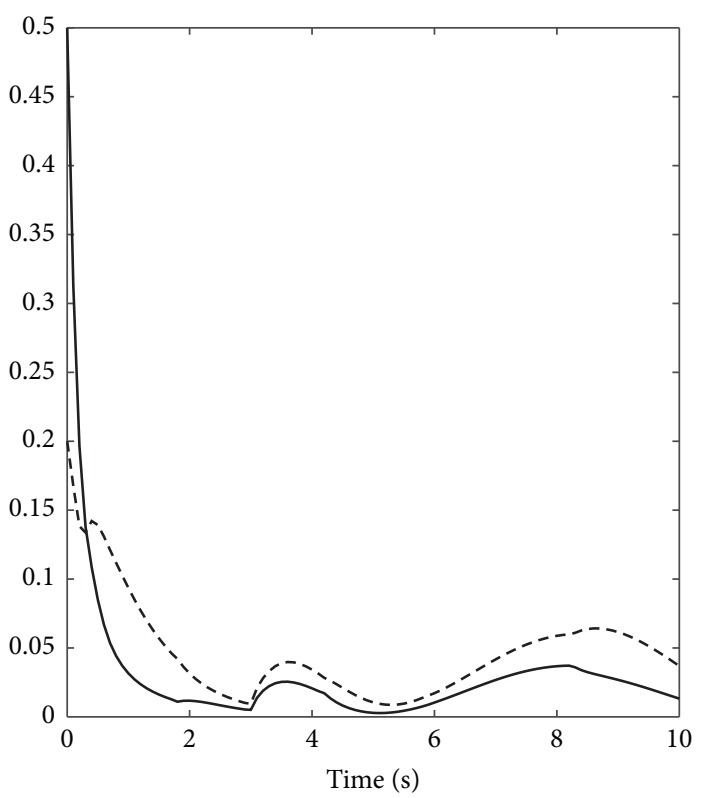

$$
\begin{array}{r}
x 1 \\
---x 2
\end{array}
$$

FIGURE 2: State trajectories of the closed-loop system (1) under static output feedback law.

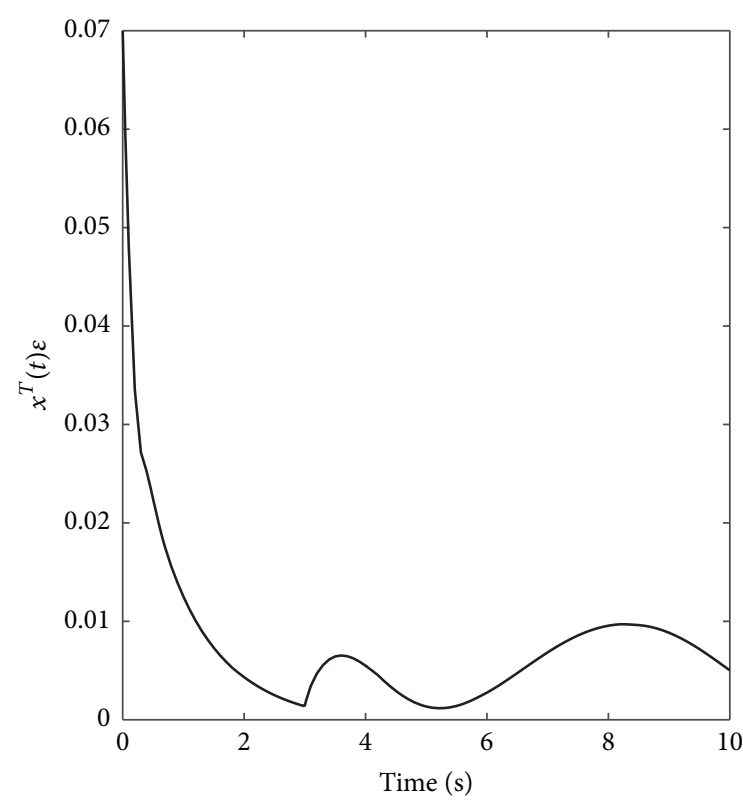

FIGURE 3: The evolution of $x^{T}(t) \varepsilon$ of system (1) under static output feedback law.

and $x(\theta)=0, \theta \in[-\tau, 0)$, which satisfy the condition $\sup _{-\tau \leq t \leq 0}\left\{x^{T}(t) \delta\right\} \leq 1$. Figure 1 depicts the switching signal with MDADT. Under the static output feedback control law, Figures 2 and 3 are obtained to show the state trajectories and the evolution of $x^{T}(t) \varepsilon$ of the closed-loop system (1). Under the state feedback control law, Figures 4 and 5 depict the 


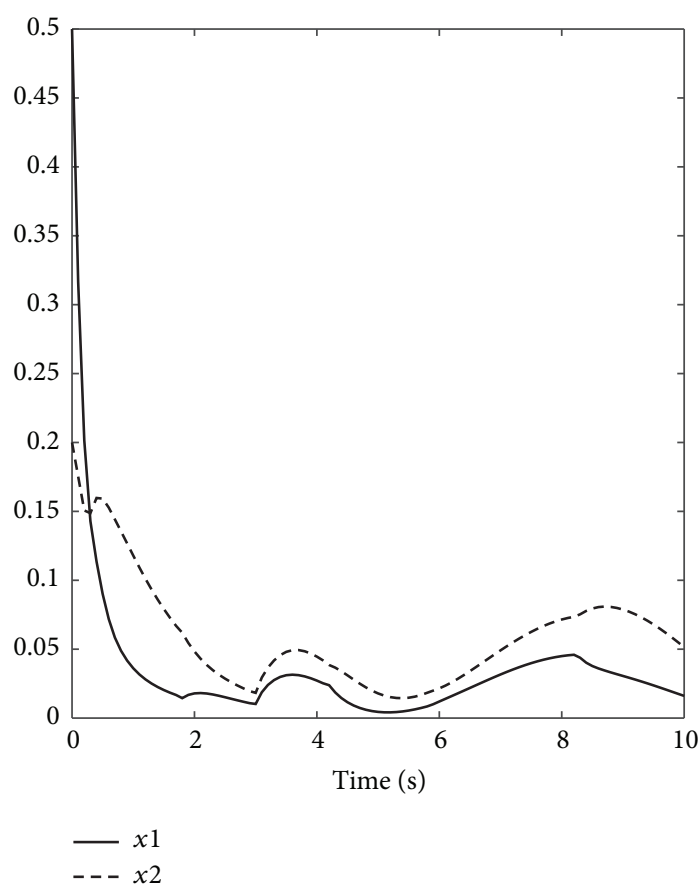

FIGURE 4: State trajectories of the closed-loop system (1) under state feedback law.

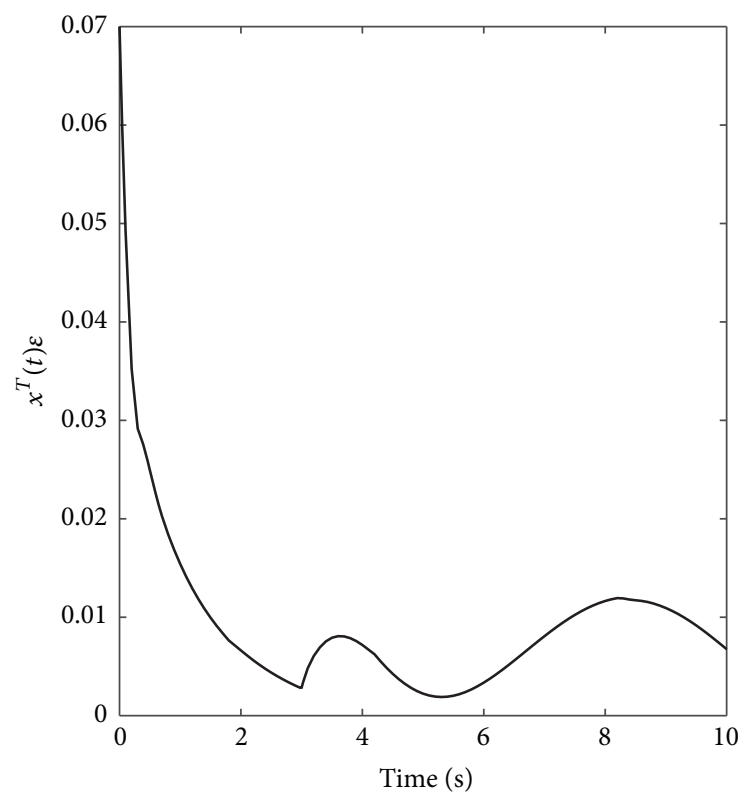

Figure 5: The evolution of $x^{T}(t) \varepsilon$ of system (1) under state feedback law.

state trajectories and the evolution of $x^{T}(t) \varepsilon$ of the closedloop system (1). The cost value is $J^{*}=50.9407$, which can be obtained by (44) or (49).

Remark 16. Reference [6] considered the problem of exponential $L_{1}$ output tracking control for positive switched linear systems with ADT, which focuses on the asymptotic stability and the exponential $L_{1}$ performance index. There are some differences between [6] and this paper. Firstly, the finite-time stability in this paper and the asymptotic stability in [6] are two absolutely different concepts. Secondly, the performance indexes are also different. Finally, this paper uses MDADT approach instead of ADT method in [6]. So we do not compare the simulation results.

\section{Conclusions}

This paper has dealt with the problem of guaranteed cost finite-time control for positive switched systems with tinevarying delays. A novel guaranteed cost performance index is introduced. Based on the MDADT approach, some sufficient conditions and two kinds of controllers are obtained to guarantee that the closed-loop system is GCFTB. Finally, a numerical example is provided to show the effectiveness of the proposed method.

\section{Competing Interests}

The authors declare that there is no conflict of interests regarding the publication of this paper.

\section{Acknowledgments}

The authors are grateful for the supports of the National Natural Science Foundation of China under Grants U1404610, 61473115, 61374077, and 61203047.

\section{References}

[1] X. Zhao, Y. Yin, and X. Zheng, "State-dependent switching control of switched positive fractional-order systems," ISA Transactions, vol. 62, pp. 103-108, 2016.

[2] S. Li and Z. Xiang, "Stability, -gain and -gain analysis for discrete-time positive switched singular delayed systems," Applied Mathematics and Computation, vol. 275, pp. 95-106, 2016.

[3] J. Zhang, X. Zhao, R. Zhang, and S. Zhou, "Dual approach to stability and stabilisation of uncertain switched positive systems," International Journal of Systems Science, vol. 48, no. 4, pp. 873-884, 2017.

[4] J. Liu, J. Lian, and Y. Zhuang, "Robust stability for switched positive systems with D-perturbation and time-varying delay," Information Sciences, vol. 369, pp. 522-531, 2016.

[5] M. Xiang and Z. Xiang, "Stability, $\mathrm{L}_{1}$-gain and control synthesis for positive switched systems with time-varying delay," Nonlinear Analysis. Hybrid Systems, vol. 9, pp. 9-17, 2013.

[6] S. Liu and Z. Xiang, "Exponential $\mathrm{L}_{1}$ output tracking control for positive switched linear systems with time-varying delays," Nonlinear Analysis: Hybrid Systems, vol. 11, pp. 118-128, 2014.

[7] M. Xiang and Z. Xiang, "Robust fault detection for switched positive linear systems with time-varying delays," ISA Transactions, vol. 53, no. 1, pp. 10-16, 2014.

[8] M. Xiang and Z. Xiang, "Observer design of switched positive systems with time-varying delays," Circuits, Systems, and Signal Processing, vol. 32, no. 5, pp. 2171-2184, 2013.

[9] J. Liu, K. Zhang, G. Pang, and H. Wei, "Controller synthesis for constrained discrete-time switched positive linear systems," Nonlinear Analysis: Hybrid Systems, vol. 19, pp. 1-12, 2016. 
[10] D. Peter, "Short time stability in linear time-varying systems," in Proceedings of the IRE International Convention Record, pp. 83-87, 1961.

[11] F. Amato, M. Ariola, and P. Dorato, "Finite-time control of linear systems subject to parametric uncertainties and disturbances," Automatica, vol. 37, no. 9, pp. 1459-1463, 2001.

[12] Z. Xiang, Y.-N. Sun, and M. S. Mahmoud, "Robust finitetime $H \infty$ control for a class of uncertain switched neutral systems," Communications in Nonlinear Science and Numerical Simulation, vol. 17, no. 4, pp. 1766-1778, 2012.

[13] X. Lin, S. Li, and Y. Zou, "Finite-time stabilization of switched linear time-delay systems with saturating actuators," Applied Mathematics and Computation, vol. 299, pp. 66-79, 2017.

[14] S. Huang and Z. Xiang, "Finite-time stabilization of switched stochastic nonlinear systems with mixed odd and even powers," Automatica. A Journal of IFAC, the International Federation of Automatic Control, vol. 73, pp. 130-137, 2016.

[15] Y. Chen, Q. Liu, R. Lu, and A. Xue, "Finite-time control of switched stochastic delayed systems," Neurocomputing, vol. 191, pp. 374-379, 2016.

[16] Y. Ma, L. Fu, Y. Jing, and Q. Zhang, "Finite-time $\mathrm{H}^{\infty}$ control for a class of discrete-time switched singular time-delay systems subject to actuator saturation," Applied Mathematics and Computation, vol. 261, pp. 264-283, 2015.

[17] L. Zhu, Y. Shen, and C. Li, "Finite-time control of discrete-time systems with time-varying exogenous disturbance," Communications in Nonlinear Science and Numerical Simulation, vol. 14, no. 2, pp. 361-370, 2009.

[18] G. Chen and Y. Yang, "Finite-time stability of switched positive linear systems," International Journal of Robust and Nonlinear Control, vol. 24, no. 1, pp. 179-190, 2014.

[19] M. Xiang and Z. Xiang, "Finite-time $L_{1}$ control for positive switched linear systems with time-varying delay," Communications in Nonlinear Science and Numerical Simulation, vol. 18, no. 11, pp. 3158-3166, 2013.

[20] J. Liu, J. Lian, and Y. Zhuang, "Output feedback $\mathrm{L}_{1}$ finite-time control of switched positive delayed systems with MDADT," Nonlinear Analysis. Hybrid Systems, vol. 15, pp. 11-22, 2015.

[21] L. Wu and Z. Wang, "Guaranteed cost control of switched systems with neutral delay via dynamic output feedback," International Journal of Systems Science, vol. 40, no. 7, pp. 717728, 2009.

[22] B. Pang, X. Liu, Q. Jin, and W. Zhang, "Exponentially stable guaranteed cost control for continuous and discrete-time Takagi-Sugeno fuzzy systems," Neurocomputing, vol. 205, pp. 210-221, 2016.

[23] X. Yang, D. Liu, Q. Wei, and D. Wang, "Guaranteed cost neural tracking control for a class of uncertain nonlinear systems using adaptive dynamic programming," Neurocomputing, vol. 198, pp. 80-90, 2016.

[24] D. Wang, D. Liu, C. Mu, and H. Ma, "Decentralized guaranteed cost control of interconnected systems with uncertainties: a learning-based optimal control strategy," Neurocomputing, vol. 214, pp. 297-306, 2016.

[25] Y. Zhao, G. Guo, and L. Ding, "Guaranteed cost control of mobile sensor networks with Markov switching topologies," ISA Transactions, vol. 58, pp. 206-213, 2015.

[26] Z. Yan, G. Zhang, J. Wang, and W. Zhang, "State and output feedback finite-time guaranteed cost control of linear itô stochastic systems," Journal of Systems Science \& Complexity, vol. 28 , no. 4, pp. 813-829, 2015.
[27] L. Zhang, X.-Y. Wang, and K. Zhang, "Non-fragile finite-time guaranteed cost fuzzy control for continuous-time nonlinear systems," International Journal of Computational Intelligence Systems, vol. 7, no. 1, pp. 129-135, 2014.

[28] L. Liu, M. Cheng, and $\mathrm{M} . \mathrm{Xu}$, " $H_{\infty}$ guaranteed cost finitetime control for stochastic differential inclusion systems," in Proceedings of the IEEE International Conference on Information and Automation (ICIA '15), pp. 1447-1451, August 2015.

[29] P. Niamsup, K. Ratchagit, and V. N. Phat, "Novel criteria for finite-time stabilization and guaranteed cost control of delayed neural networks," Neurocomputing, vol. 160, pp. 281-286, 2015. 


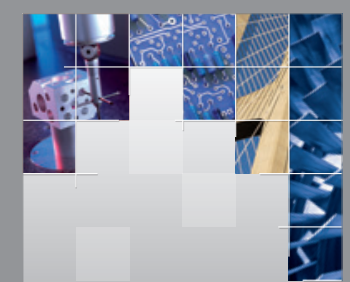

\section{Enfincering}
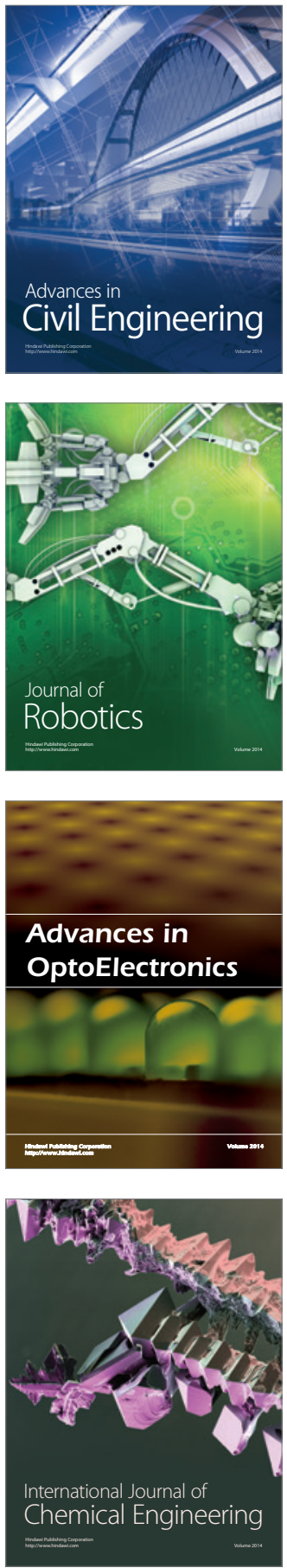

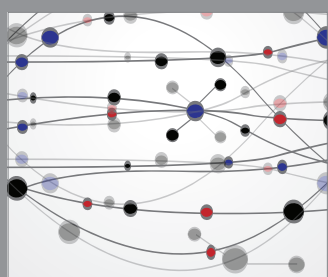

The Scientific World Journal

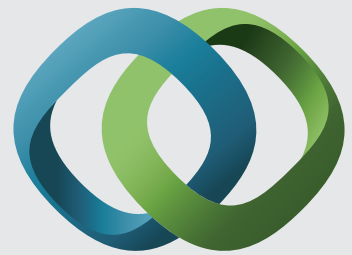

\section{Hindawi}

Submit your manuscripts at

https://www.hindawi.com
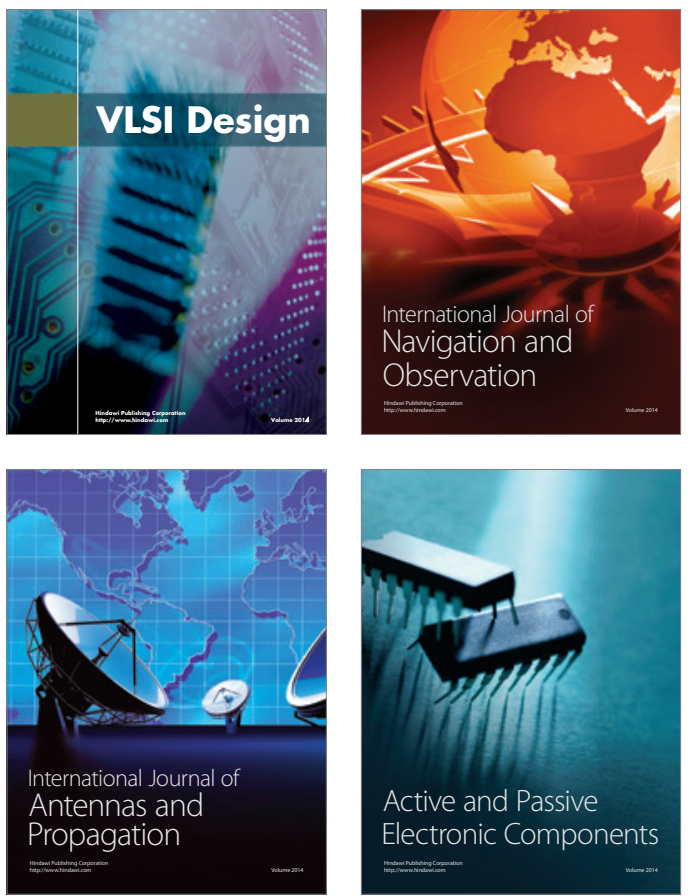
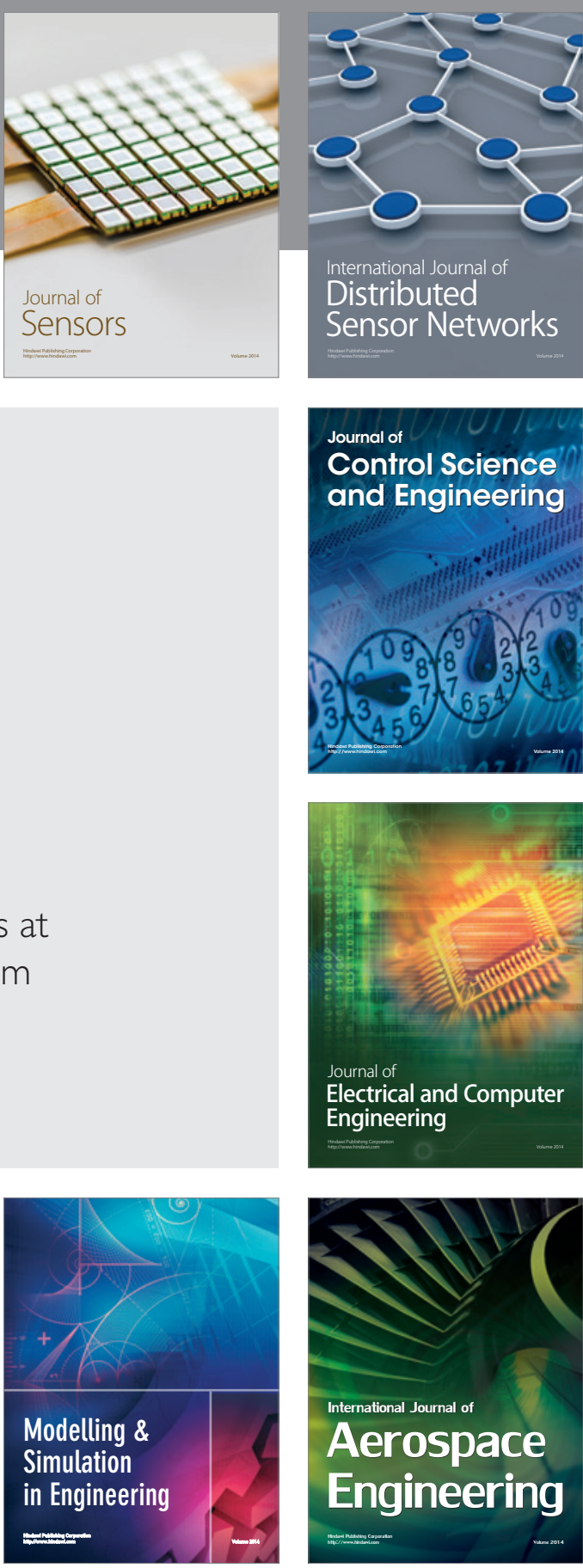

International Journal of

Distributed

Sensor Networks

$-$

Joumal of

Control Science

and Engineering
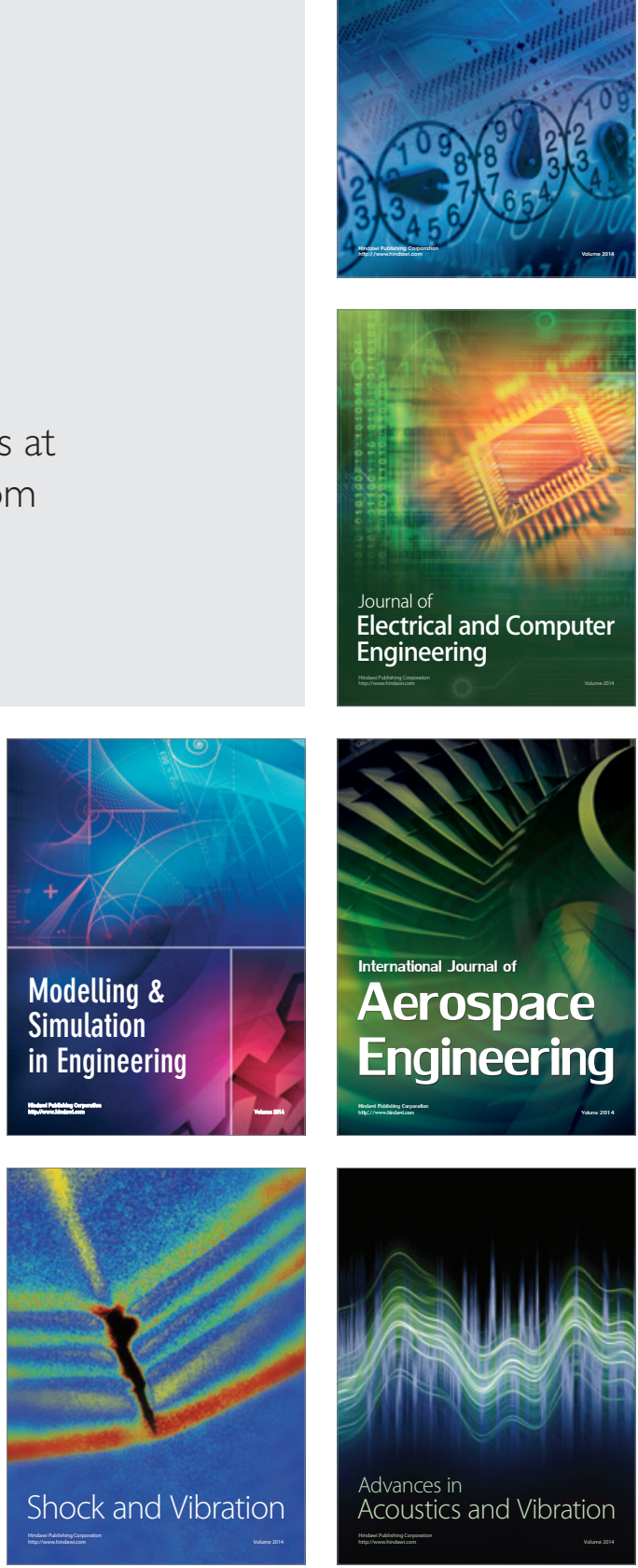